\title{
Assessment and Validation of VLES Turbulence Models for Cavitating Flow
}

\author{
Wei Guan ${ }^{1,2}$, Zhixia He ${ }^{\star 1}$, Liang Zhang ${ }^{2}$, Xianyin Leng ${ }^{1}$, Zhuang Shao ${ }^{3}$ \\ ${ }^{1}$ Institute for Energy Research, Jiangsu University, Zhenjiang, China \\ ${ }^{2}$ School of Energy and Power Engineering, Jiangsu University, Zhenjiang, China \\ ${ }^{3}$ Shandong Institute of Advanced Technology, Chinese Academy of Sciences, Shandong, \\ China \\ ${ }^{*}$ Corresponding author email : zxhe@ujs.edu.cn
}

\begin{abstract}
Cavitating flow has been widely studied in many application fields. This paper aims to compare the results of three turbulence models for the simulation of cavitating flows; one is the newly developed VLES (Very-large eddy simulation) model, another is SST k-omega turbulence model and the last one is a LES (Large eddy simulation) models including the WALE (Walladapting local eddy viscosity model). The VLES model is a self-adaptive turbulence modelling method which can resolve the turbulence based on the local mesh resolution and the local turbulence scale information. All models are compared in an U-type throttle nozzle and an experimental injector nozzle. The study demonstrates that the VLES model can provide more accurate results of cavitation than the SST k-omega model, even not lose to the LES model. The results, based on a series of the grid with different resolutions, prove that the VLES model can provide acceptability predictions of cavitating flow on relatively coarse meshes.
\end{abstract}

\section{Keywords}

Very large eddy Simulation; String cavitation; Cloud cavitation; Large eddy simulation.

\section{Introduction}

Cavitation is considered as one of the crucial phenomena of internal flow inside diesel injector nozzle holes. The geometry-induced cavitation generally originates at the upper and lower lips of the nozzle hole entrance, and its occurrence and development mostly rely on the injection pressure and the geometry of the nozzle [1-3]. The geometry-induced cavitation regime has been well-understood to promote the primary break-up and the perturbations of the spray plume downstream the nozzle exit. Besides, it also significantly reduces the nozzle discharge coefficient and inevitably causes undesired surface erosion [4-6]. The vortex-induced cavitation with normally string type is correspondingly also called string cavitation. Some investigations [7-10] indicated that there was a significant improvement of spray atomization downstream in the existence of string cavitation, particularly a prominently increasing in spray angle.

The interaction of turbulence and cavitation has been widely studied on various application fields using numerical tools. Turbulence models in the field of Computational Fluid Dynamics (CFD) can be roughly divided into three major categories, i.e. Reynolds Averaged NavierStokes (RANS), Large Eddy Simulation (LES), and Direct Numerical Simulation (DNS), according to the decreasing level of turbulence modelling. Several RANS simulations with various turbulence closure models, such as $k-\varepsilon$ model and k-omega model, show poor performance in predicting a re-entrant jet motion and the cavity shedding pattern [11-13]. The RANS models are reported to fail to predict pressures below the saturation pressure, while the LES model results can reproduce an unsteady flow field and some small vortices [14]. The LES model can accurately resolve the large scale coherent turbulent scales, but it is computationally more expensive than RANS models. In terms of DNS, it needs very fine mesh resolution and its cost becomes prohibitive. With the development of computer technology, 
the LES model is becoming a prevailing turbulent method for predicting cavitating flow in some recent studies [15-17].

In recent years, a new self-adaptive turbulence model, namely VLES (Very-Large Eddy Simulation) [18-20], is developed by Han et al. as the hybrid RANS-LES modelling to reproduce the complicated turbulent flow in many application fields. The VLES method is an unified simulation approach enabling a seamless evolution from RANS to LES and finally approaching DNS depending on the numerical resolution.

The aim of this work is to examine the influence of various turbulence models, including SST k-omega, LES and VLES, on the prediction of cavitating flow in an U-type throttle nozzle and an injector nozzle.

\section{Description of the CFD approach}

The simulations were performed using the commercial CFD code ANSYS Fluent. A two-phase volume of fluid (VOF) model is used to solve the internal nozzle flow. All multiphase components share the same pressure and velocity at any given locations. The prevailing model to describe the cavitation phase change process is to establish the transport relationship between liquid and vapor phases based on the Rayleigh-Plesset equations [21]. ZwartGerber-Belamri (ZGB) [22] cavitation model was used to calculate and describe the growth and collapse of one single bubble in the liquid. The final expressions of the cavitation model are as follows:

$\dot{R}_{e}=F_{v a p} \frac{3 \alpha_{n u c}\left(1-a_{v}\right) \rho_{v}}{R_{B}} \sqrt{\frac{2}{3} \frac{\left(p_{v}-p\right)}{\rho_{l}}}, p \leq p_{v}$

$\dot{R}_{c}=F_{\text {cond }} \frac{3 \alpha_{v} \rho_{v}}{R_{B}} \sqrt{\frac{2}{3} \frac{\left(p-p_{v}\right)}{\rho_{l}}}, p>p_{v}$

where $F_{\text {vap }}$ and $F_{\text {cond }}$ are empirical coefficients. $\rho_{/}$and $\rho_{v}$ are the density of liquid and vapor respectively. $p$ is the local pressure and $p_{v}$ is the vapor pressure. $R_{B}$ represents the mean radius of bubbles, which is assumed to be $0.001 \mathrm{~mm}$.

The SST k-omega model is a two-equation turbulence model, which solves for turbulent kinetic energy $(k)$ and specific dissipation rate (omega). It combines the advantages of the standard k-omega model and the $k-\varepsilon$ model. In LES model, large eddies are resolved directly, while small eddies are modelled. The Wall Adapted Local Eddy- viscosity (WALE) model is applied to simulate the nozzle cavitating flow. It allows the correct treatment of laminar zones in the domain. Both turbulence models have been introduced in many research works.

In the present study, the VLES model is blended with the BSL k-omega turbulence model, which can be called VLES BSL k-omega model. In VLES model, the form of the underlying BSL $k$-omega model is not changed, and only to modify the turbulent viscosity by scaling with a resolution control function $F_{r}$. The form of this parameter is given as follow,

$F_{r}=\operatorname{MIN}\left[1.0,\left(\frac{1.0-\exp \left(-\beta L_{c} / L_{k}\right)}{1.0-\exp \left(-\beta L_{i} / L_{k}\right)}\right)^{2}\right]$

$L_{c}=C x(\Delta x \Delta y \Delta z)^{1 / 3}, L_{i}=k^{\frac{3}{2}} /\left(\beta^{*} k \omega\right), L_{k}=v^{\frac{3}{4}} /\left(\beta^{*} k \omega\right)^{1 / 4}$

where $\beta$ is $0.002, C_{x}$ is 0.61 and $\beta^{*}$ is $0.09 . L_{c}, L_{i}$, and $L_{k}$ are the turbulent cut-off length scale, integral length scale, and Kolmogorov length scale, respectively. The turbulent viscosity is scaled by the resolution control function $F_{r}$.

$\mu_{t}=F_{r} * \rho k / \omega$ 
The details of the VLES model have been reported in [18-20] and will not be repeated here. In this work, the convective terms are discretized using a central differencing scheme. The second-order upwind scheme is used for the turbulence model equations, and a second-order implicit scheme is used for the temporal discretization. The SIMPLE algorithm is used for pressure-velocity coupling. The CFL number is 0.9 .

\section{Results and Discussion}

The simulation cases of cavitating flow is implemented in an U-type throttle nozzle referred in the research of Winklhofer [23]. The pressure of the fluid in the upstream is held constant at 100 bar while the downstream pressure is controlled for various cases starting from 80 bar to 15 bar. The nozzle geometry is depicted in Figure 1. The tunnel geometry shape is a $0.3 \mathrm{~mm}$ thick sheet between two flat transparent plates, with $0.301 \mathrm{~mm}$ inlet height, $0.284 \mathrm{~mm}$ outlet height, $0.02 \mathrm{~mm}$ entrance corner radius, and $1 \mathrm{~mm}$ throttle hole length. A total hexahedral grid of 560,000 is generated by ICEM. The hole zone is refined to capture flow characteristics, where the maximum grid size is $6 \mu \mathrm{m}$ near the wall and $10 \mu \mathrm{m}$ in the middle area.
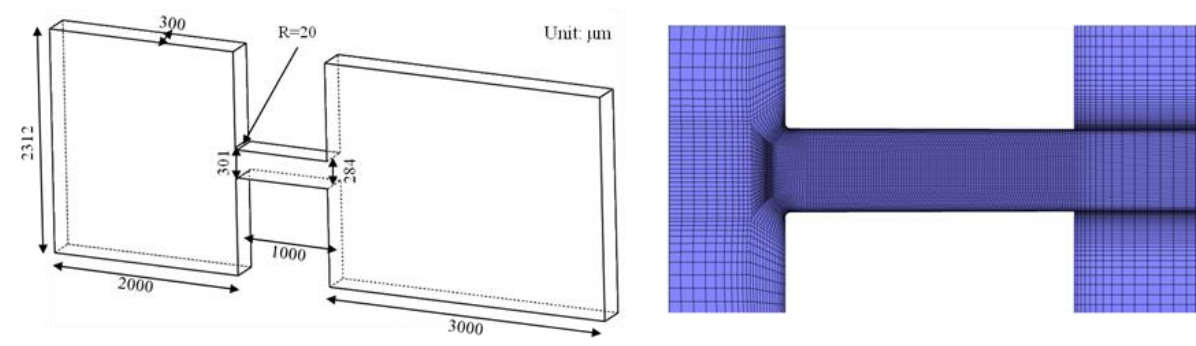

Figure 1. The schematics of the nozzle. Left: geometrical nozzle, right: detailed mesh model.

\begin{tabular}{|c|c|c|c|c|}
\hline Mesh number & Experiment data & SST k-omega & LES & VLES BSL k- $\omega$ \\
\hline 1 million & & 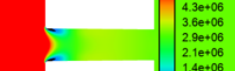 & & 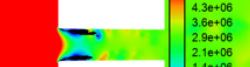 \\
\hline 2 million & & & & 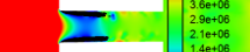 \\
\hline 2.5 million & & & & \\
\hline 3 million & & & & 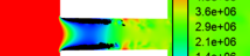 \\
\hline
\end{tabular}

Figure 2. Comparison of total void fraction between (i) experimental data and (ii) time-averaged predictions of vapor distribution (black region) at 0.1 vapour volume fraction with three turbulence models under different mesh resolution. Mesh resolution evaluation of LES quality metric is also shown according to the work of Celik et al.[24].

In order to assess the comparisons of three turbulence models by means of capturing the flow conditions within the U-type throttle nozzle, model predictions of the vapor distribution within 0.1 vapour volume fraction are compared to the extent of cavitation as indicated in the 
experimental images of Winklhofer [23]. A series of representative comparisons are shown in Figures 2 for a pressure drop condition of 70 bar, where both the experimental and computational images have been time-averaged after steady state conditions have been reached. Under this case, cavitation extends along the wall boundary until approximately half of the length of the throttle.

As can be seen from the figure, the LES model and VLES model can predict the cavitation extent accurately. SST k-omega model hardly reproduced the cavitation development. Cavitating flow itself has the highly transient characteristics including the shedding and reproduction of cavitation bubbles. The Reynolds Averaged method may hardly capture the cavitation patterns, but the adjustment of empirical parameters of the cavitation models probably can resolve it. The VLES model can provide more accurate results of cavitation than the SST k-omega model, even not lose to the LES model. It can be concluded the contours of VLES results are closer to the real physical flow field. As mentioned, VLES model is a selfadaptive turbulence modelling method which can resolve the turbulence based on the local mesh resolution and the local turbulence scale information. Therefore, the comparisons of different mesh resolutions with VLES model are shown in Figure 2. The VLES model can present the cavitation pattern similar to the experimental image at a grid of two million, though less cavitation is predicted at a grid of one million. Therefore, the VLES model can provide acceptability predictions of cavitating flow on relatively coarse meshes. Based on the results, the capability prediction of cavitation in diesel injector nozzles by VLES can be assessed in the following part.

The simulated injector nozzle replicates the geometrical topology of the real-size injector tip used in the experiment for the better model validation, as shown in Figure 3 [25]. The two holes of this nozzle are slightly tapered for the suppression of geometry-induced near-wall sheet cavitation and cloud cavitation, and then the clear presence of the individual string cavitation in the injector nozzle holes. Furthermore, a back pressure chamber is added downstream the hole exit in order to move the outlet boundary condition away from the areas of interest. High-quality hexahedra-dominant cells are produced for the most parts of the injector nozzle geometry, except for a small region in the SAC chamber beneath the needle tip head where is meshed by unstructured tetrahedral cells. Grid refinements of sufficient resolution in the SAC chamber and the central area of the hole are paid more attention, for considering the sensitive area of string cavitation development.

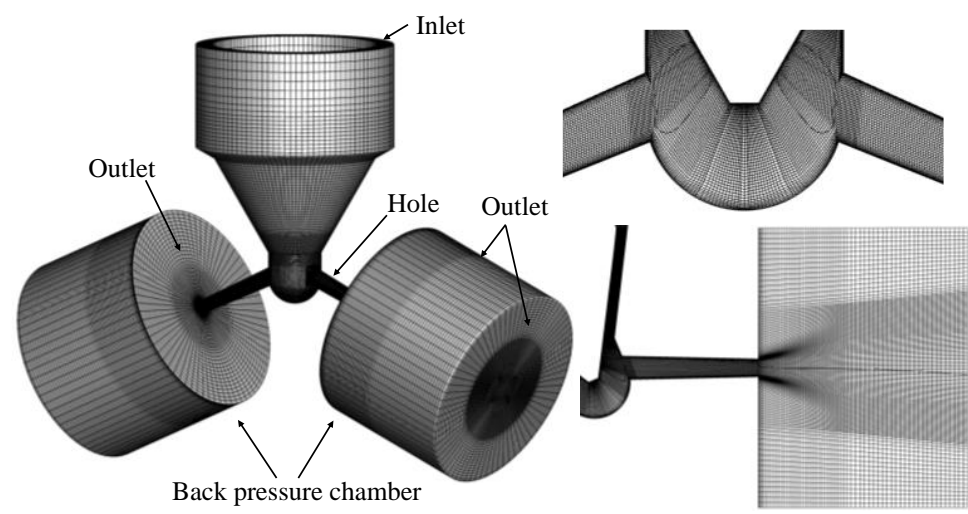

Figure 3. The schematics of the experimental injector nozzle [25]. 


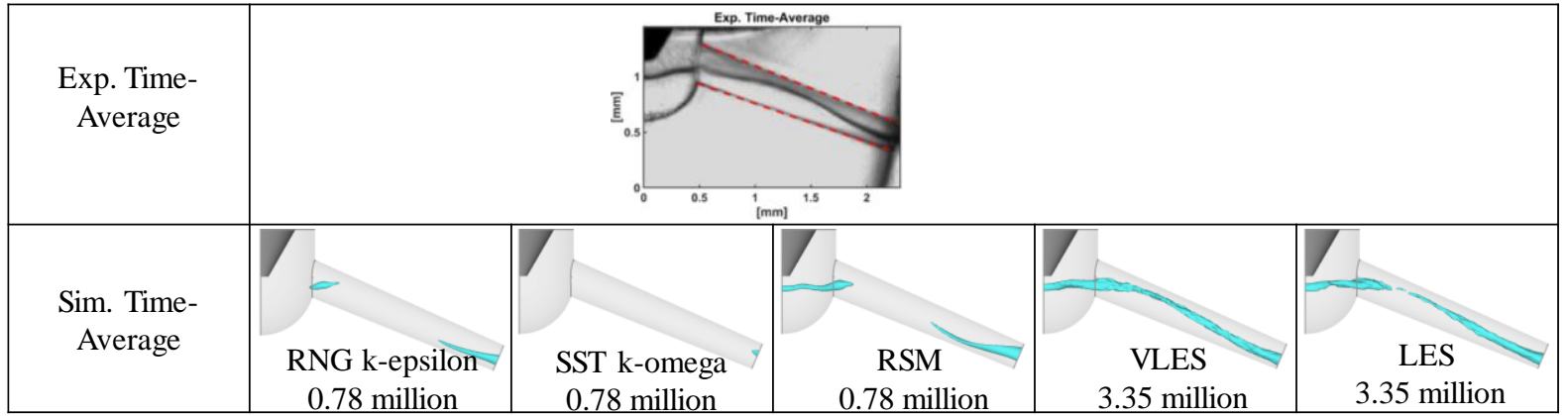

Figure 4. Comparison of total void fraction between (i) experimental data and (ii) time-averaged predictions of vapor distribution at 0.1 vapour volume fraction with different turbulence models under different mesh resolution.

As can be seen from the figure, the LES model and VLES model can predict the string cavitation morphology accurately which is similar to the experimental data. The string cavitation stably exists in the SAC chamber and nozzle hole, although there is a little breakage in the middle part of nozzle hole. This kind of breakage is more obvious in the simulation of RSM model. However, SST k-omega model hardly reproduced the string cavitation morphology and there is only a little cavitation appearing at the hole exit, because the Reynolds Averaged method may hardly capture the cavitation patterns. Similarly, the VLES model can provide more accurate results of cavitation than the SST k-omega model, even not lose to the LES model. It can be concluded the contours of VLES results are closer to the real physical flow field. Moreover, the VLES model can provide acceptability predictions of cavitating flow on relatively coarse meshes which is only half of the grid number in large eddy simulation.

\section{Conclusions}

1. The VLES model can provide more accurate results of cavitation than the SST KOmega model, even not lose to the LES model.

2. The VLES model can provide acceptability predictions of cavitating flow on relatively coarse meshes.

\section{Acknowledgments}

This research was supported by the National Natural Science Foundation of China (No.51776088), the National Key Research and Development Project of China (2019YFB1504004)\&(2016YFD0700602), High-tech Research Key laboratory of Zhenjiang (SS2018002) and Research Innovation Plan for Postgraduates in Jiangsu Universities of China (KYCX20_3023).

\section{References}

[1] He, Z., Shao, Z., Wang, Q., Zhong, W., and Tao, X., 2015, "Experimental study of cavitating flow inside vertical multi-hole nozzles with different length-diameter ratios using diesel and biodiesel," Experimental Thermal and Fluid Science, 60, pp. 252-262.

[2] Salvador, F., De la Morena, J., Martínez-López, J., and Jaramillo, D., 2017, "Assessment of compressibility effects on internal nozzle flow in diesel injectors at very high injection pressures," Energy Conversion and Management, 132, pp. 221-230.

[3] Sou, A., Biçer, B., and Tomiyama, A., 2014, "Numerical simulation of incipient cavitation flow in a nozzle of fuel injector," Computers \& Fluids, 103, pp. 42-48.

[4] Arabnejad, M. H., Svennberg, U., and Bensow, R. E., 2021, "Numerical assessment of cavitation erosion risk using incompressible simulation of cavitating flows," Wear, 464, p. 203529.

[5] Cristofaro, M., Edelbauer, W., Koukouvinis, P., and Gavaises, M., 2020, "A numerical study on the effect of cavitation erosion in a diesel injector," Applied Mathematical Modelling, 78, pp. 200-216. 
[6] Molina, S., Salvador, F., Carreres, M., and Jaramillo, D., 2014, "A computational investigation on the influence of the use of elliptical orifices on the inner nozzle flow and cavitation development in diesel injector nozzles," Energy conversion and management, 79, pp. 114-127.

[7] Andriotis, A., and Gavaises, M., 2009, "Influence of vortex flow and cavitation on near-nozzle diesel spray dispersion angle," Atomization and Sprays, 19(3).

[8] Hayashi, T., Suzuki, M., and Ikemoto, M., "Visualization of internal flow and spray formation with real size diesel nozzle," Proc. 12th Triennial International Conference on Liquid Atomization and Spray Systems, ICLASS, pp. 26.

[9] Pratama, R. H., Sou, A., Katsui, T., and Nishio, S., 2017, "String cavitation in a fuel injector," Atomization and Sprays, 27(3).

[10] Watanabe, H., Nishikori, M., Hayashi, T., Suzuki, M., Kakehashi, N., and Ikemoto, M., 2015, "Visualization analysis of relationship between vortex flow and cavitation behavior in diesel nozzle," International Journal of Engine Research, 16(1), pp. 5-12.

[11] Coutier-Delgosha, O., Fortes-Patella, R., and Reboud, J.-L., 2003, "Evaluation of the turbulence model influence on the numerical simulations of unsteady cavitation," J. Fluids Eng., 125(1), pp. 38-45.

[12] Coutier-Delgosha, O., Reboud, J., and Delannoy, Y., 2003, "Numerical simulation of the unsteady behaviour of cavitating flows," International journal for numerical methods in fluids, 42(5), pp. 527-548.

[13] Dular, M., and Coutier-Delgosha, O., 2009, "Numerical modelling of cavitation erosion," International journal for numerical methods in Fluids, 61(12), pp. 1388-1410.

[14] Koukouvinis, P., Naseri, H., and Gavaises, M., 2017, "Performance of turbulence and cavitation models in prediction of incipient and developed cavitation," International Journal of Engine Research, 18(4), pp. 333-350.

[15] Guo, G., He, Z., Chen, Y., Wang, Q., Leng, X., and Sun, S., 2017, "LES investigations on effects of the residual bubble on the single hole diesel injector jet," International Journal of Heat and Mass Transfer, 112, pp. 18-27.

[16] Koukouvinis, P., Gavaises, M., Li, J., and Wang, L., 2016, "Large Eddy Simulation of Diesel injector including cavitation effects and correlation to erosion damage," Fuel, 175, pp. 26-39.

[17] Trummler, T., Schmidt, S. J., and Adams, N. A., 2020, "Investigation of condensation shocks and re-entrant jet dynamics in a cavitating nozzle flow by Large-Eddy Simulation," International Journal of Multiphase Flow, 125, p. 103215.

[18] Han, X., and Krajnović, S., 2013, "An efficient very large eddy simulation model for simulation of turbulent flow," International Journal for Numerical Methods in Fluids, 71(11), pp. 1341-1360.

[19] Han, X., and Krajnović, S., 2015, "Very-large-eddy Simulation based on k-w model," AIAA Journal, 53(4), pp. 1103-1108.

[20] Tiwari, P., Xia, Z., and Han, X., 2020, "Comparison of VLES and LES Turbulence Modeling for Swirling Turbulent Flow," Journal of Applied Fluid Mechanics, 13(4), pp. 1107-1116.

[21] Brennen, C. E., 2014, Cavitation and bubble dynamics, Cambridge University Press.

[22] Zwart, P. J., Gerber, A. G., and Belamri, T., "A two-phase flow model for predicting cavitation dynamics," Proc. Fifth international conference on multiphase flow, Yokohama, Japan.

[23] Winklhofer, E., Kull, E., Kelz, E., and Morozov, A., "Comprehensive hydraulic and flow field documentation in model throttle experiments under cavitation conditions," Proc. Proceedings of the ILASS-Europe conference, Zurich, pp. 574-579.

[24] Celik, I., Cehreli, Z., and Yavuz, I., 2005, "Index of resolution quality for large eddy simulations."

[25] Guan, W., He, Z., Zhang, L., Guo, G., Cao, T., and Leng, X., 2021, "Investigations on interactions between vortex flow and the induced string cavitation characteristics in real-size diesel tapered-hole nozzles," Fuel, 287, p. 119535. 